\title{
Can Dips of Boyajian's Star be Explained by Circumsolar Rings?
}

\author{
J. I. Katz, ${ }^{1 \star}$ \\ ${ }^{1}$ Department of Physics and McDonnell Center for the Space Sciences, Washington University, St. Louis, Mo. 63130 USA
}

29 August 2018

\begin{abstract}
Could the dips of "Boyajian's Star" (KIC 8462852) have been caused by matter in our Solar System? The interval between periods of deep dips is nearly twice the orbital period of the Kepler satellite. I consider heliocentric obscuring rings in the outer Solar System that graze the line of sight to the star once per Kepler orbit. The hypothesis predicts that future dips may be observed from Earth during windows separated by a year, although their detailed structure depends on the distribution of particles along the ring. Dips observed at terrestrial sites separated by $>1000 \mathrm{~km}$ in a direction perpendicular to the Earth's orbital motion may be uncorrelated.
\end{abstract}

Key words: Kuiper belt: general, stars: peculiar, (stars:) planetary systems

\section{INTRODUCTION}

"Boyajian's star" (KIC 8462852) is a peculiar variable discovered by the Kepler exoplanet-finding mission (Borucki, et al. 2010; Koch, et al. 2010). Boyajian et al. (2016); Wright \& Sigurðsson (2016) review these and other observations and contain extensive references to the literature. Spectroscopically an ordinary F3V dwarf, Boyajian's star shows remarkable deep dips. These are much too deep (up to 21\%) to have been caused by planetary transits, for the radius of the transiting objects would have to be nearly half $(\sqrt{0.21} \times)$ the radius of the F3V star, or at least $5 \times 10^{10}$ $\mathrm{cm}$. This is several times larger than the radius of any cold object, or even than the radii of "hot jupiters". Planetary transits are also excluded because the dips are not periodic.

This behavior has been interpreted (Boyajian et al. 2016) as the result of obscuration near Boyajian's star by dust clumps bound to multiple planetary objects or the transient result of breakup of comet-like objects. The clumps would not only have to be opaque but to have at least half the diameter of the star. This explanation is constrained by the absence of an infrared excess that would be produced by warm dust, and leaves many questions unanswered (Boyajian et al. 2016). Other models that place the obscuring matter close to the star, such as a nearly edge-on disc with eruptions that interpose absorbing or scattering matter in our line of sight, are also difficult to reconcile with all the data and with our understanding of their dynamics (a disc eruption would put disc matter in an inclined orbit, and would lead to periodic obscuration, mimicking a planet).

A single $16 \%$ dip several days long, but with half width

* E-mail katz@wuphys.wustl.edu at half minimum (HWHM) about 0.5 day, is separated from a complex cluster of dips, including dips of $3 \%, 8 \%$ and $21 \%$, by about 750 days. This interval is close to twice the $P_{K}=$ 372.53 d orbital period of the Kepler satellite (Borucki, et al. 2010; Koch, et al. 2010; Wright \& Sigurðsson 2016), which hints at a Solar System origin.

The dips qualitatively resemble the occultations of stars by planetary rings that led to the discovery of the rings of Uranus (Elliot, Dunham \& Mink 1977) and then of all the major planets. Here I consider the hypothesis that the matter obscuring Boyajian's star is not orbiting that star, but rather is found in the Solar System, so that the motion of the observer introduces an annual parallax and periodicity (Wright \& Sigurðsson 2016). I suggest that the obscuring matter orbits on heliocentric rings. The distribution of matter along these rings is likely patchy rather than continuous, observationally constrained by the occurrences, shapes and widths of the dips. If this is correct, then the dips of Boyajian's star are exemplars of the occultation astronomy suggested by Dyson (1992).

In this picture Boyajian's star is unique because its ecliptic latitude aligns it with matter in the remote outer Solar System, and specifically with the direction of tangency to orbits of this distant matter. Tangency increases the likelihood and prolongs the duration of deep absorption. The observed deep dips have durations of several hours that cannot be explained by non-tangent passages through narrow rings, while broad rings are excluded by the fact that only one of $\approx 150,000$ Kepler stars shows such dips. 

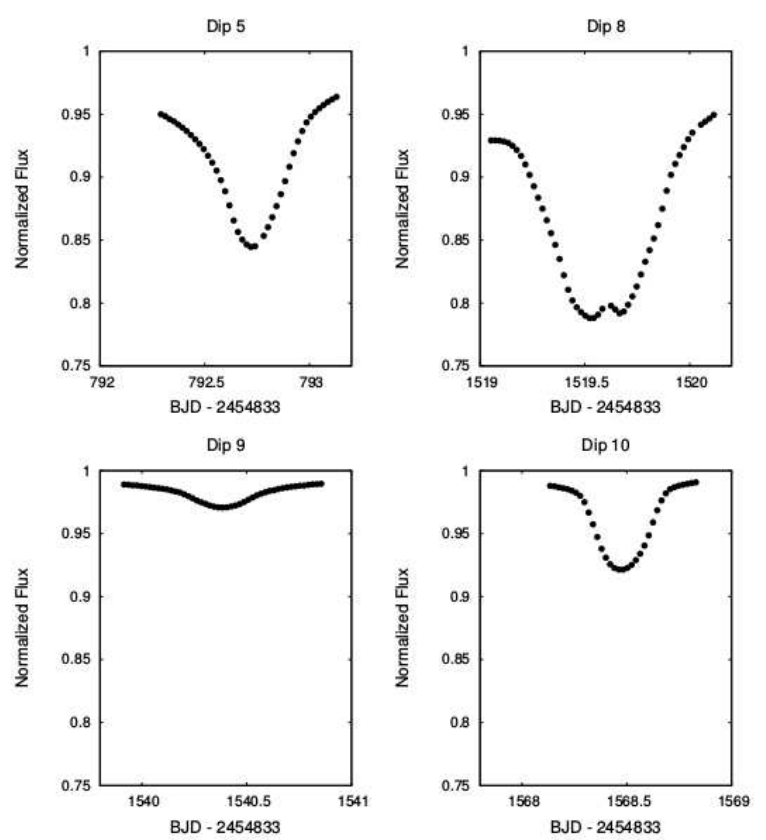

Figure 1. The four deep (>1\%) numbered dips of Boyajian et al. (2016), all plotted on the same scale to facilitate comparison. These dips have comparable, but not equal, widths, and dip 8 can be described as two overlapping narrower dips. Single data are missing near the minima of dips 5 and 8 and near day 1520.0 in dip 8 (Borucki, et al. 2010; Koch, et al. 2010). ${ }^{2}$

\section{THE DIPS}

Fig. 1 shows the deep dips in greater detail than in Boyajian et al. (2016). The dips are smooth on the data sampling time scale of $0.02 \mathrm{~d}$ and have comparable, but not identical, widths. The deepest dip (8), with a double minimum, may be the closest to exact tangency (see Sec. 5 for a discussion).

If the geometry were known exactly, the radial distribution of absorption within a ring defined by the particles' orbits could be deconvolved from the shapes of the dips. The fact that their shapes, HWHM, depths and the comparative strengths of their shoulders and penumbræ differ indicate that tangency is (unsurprisingly) not exact, precluding such deconvolution. The properties of a ring may also vary along its length, like the rings filled by disrupted cometary material that produce meteor showers.

Boyajian et al. (2016) Fourier transformed the data with the dips excised, and in the lower frequency part of the spectrum discovered a $0.8797 \mathrm{~d}$ periodicity attributed to the rotation of the star. A Fourier transform of the raw data is shown in Fig. 2. Bumps or shoulders at low frequencies reflect the rotational frequency and harmonics found by Boyajian et al. (2016). The bump around a frequency of 5/d reflects the HWHM of $\sim 0.25 \mathrm{~d}$ of the dips shown in Fig. 1 (inspection of the data shows that the other numbered dips have comparable widths). There is little or no evidence for narrower dips or other higher frequency temporal structure,

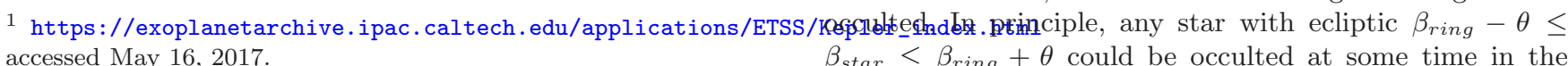
accessed May 16, 2017.
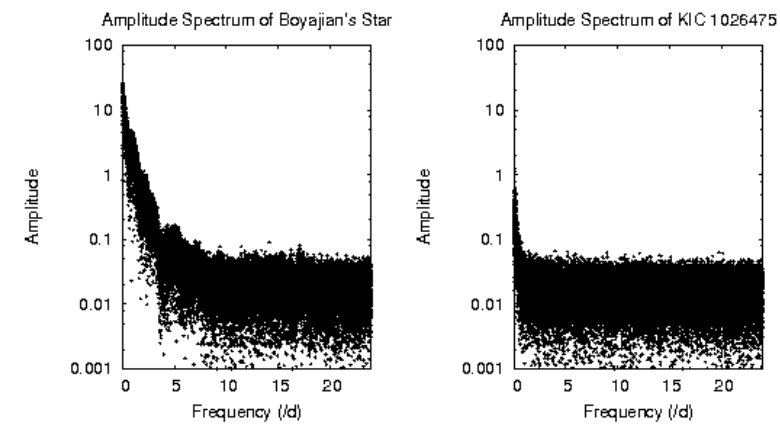

Figure 2. The Fourier transforms of the raw data records of Boyajian's Star and KIC 1026475 from BJD-2454833 = 251.8051591.001 are shown up to the Nyquist frequency. KIC 1026475 resembles Boyajian's star in magnitude and $T_{\text {eff }}$ and shows the intrinsic noise in the Kepler data. The first 131 days of the records are excluded in order to have exactly $2^{16}=65536$ evenly spaced measurement intervals for the Fast Fourier Transform. The 5885 missing data are linearly interpolated. The rotation frequency of Boyajian's star and its second and third harmonics are visible as shoulders at $1.137 / \mathrm{d}, 2.274 / \mathrm{d}$ and $3.410 / \mathrm{d}$, respectively (Boyajian et al. 2016). The bump around 5/d reflects the deep dips with HWHM $\sim 0.25 \mathrm{~d}$. The fluctuation spectrum above that frequency is almost entirely instrumental.

up to the Nyquist frequency of $24 /$ d. Comparison to the Fourier transform of the brightness of KIC 1026475, a star very similar to Boyajian's except for its temporal stability, shows essentially identical amplitudes above a frequency of $7 / \mathrm{d}$ except for the slight bumps around 9/d, 12/d and 17/d visible in Fig. 2; the remainder of the fluctuation amplitude at frequencies $>7 / \mathrm{d}$ is intrinsic to Kepler.

\section{A RING}

Boyajian's star is at a distance of about 454 pc (Boyajian et al. 2016). In the outer Solar System at a distance (from us) of $A \mathrm{AU}$ the cone of rays from the stellar disc received by the Kepler telescope (or a terrestrial telescope) has a radius of only $5 \times 10^{-5} A_{5000}$ of the radius of the star, or about $60 A_{5000} \mathrm{~km}$, where $A_{5000} \equiv A / 5000$. An obscuring object that size would be sufficient geometrically to block all the light, but briefly, although for smaller values of $A$ (such as $A \sim 50$ in the Kuiper belt) diffraction would be important and the blockage would be lengthened and shallower (Dyson 1992).

The orbital motion of Kepler (or the Earth) requires an obscuring cloud to extend along the ring a distance equal to to the distance the telescope travels (with a small correction for the motion of the obscuring matter) during the width of the deep part of a dip $\delta t \sim 0.25 \mathrm{~d}$, or $v_{\text {or } b} \delta t \sim 6 \times 10^{10}$ $\mathrm{cm}$, where $v_{\text {orb }} \approx 3 \times 10^{6} \mathrm{~cm} / \mathrm{s}$ is the difference between the observer's and the ring's orbital velocities. Penumbræ of $\sim 10^{12} \mathrm{~cm}$ are required to explain the shallower broad dips. Rather than a single object, there must be a cloud of co-moving fragments, extended along the orbit.

The geometry is shown in Fig. 3. The ring is unevenly 


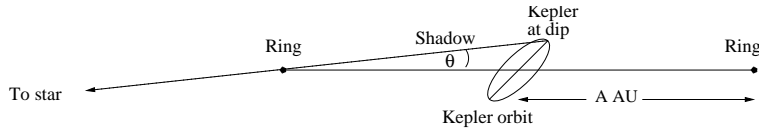

Figure 3. Nominal geometry of Boyajian's Ring, not to scale. The angle $\theta \approx(1 / A) \sin \beta_{\text {star }}$, where $A$ is the ring's semi-major axis (taken as the radius of a circular orbit) in units of the radius of the observing platform's orbit (AU for Earth, 1.0132 AU for Kepler) and $\beta$ denotes Kepler-ecliptic latitude. The ring's cross-section is smaller than can be shown to scale, neither its cross-section nor its orbit need be circular, and the distribution of debris must be patchy. In the figure the shadow is aligned with the upper extreme of the observer's orbit; analogous results would be obtained if the ring had a slightly higher ecliptic latitude so that its shadow would be aligned with the lower extreme of the observer's orbit.

observer's (Kepler's) orbit by matter in the ring, but unless the star is very close to the bounds of this inequality the passage through the ring's shadow will be rapid and the probability of detection small.

Fig. 4 shows the dependence of the declination of the ring at the right ascension of the star (in Kepler-ecliptic coordinates or in ecliptic coordinates for an Earth-based observer) as a function of the observer's mean anomaly, proportional to time for a circular ring and circular orbit of the observer. The J2000.0 ecliptic coordinates of Boyajian's star are $\lambda=323.2111^{\circ}$ and $\beta=62.1898^{\circ}$. The sinusoidal curves show the two possible ring paths (for each value of $A$ ) that maximize the time the line of sight to the star passes through the ring, and hence maximizes the probability of dips. An additional curve shows how a double dip (Sec. 5 may be produced by paths that are not exactlyi tangential. Paths further from tangency produce shorter dips, generally limited to a single Kepler datum, as estimated in the Discussion.

\section{RING PARAMETERS}

Several different estimates of the thickness of the ring can be made. These might indicate that there are several subrings.

If an obscuring event has HWHM $\delta t$, assumed to be centered around the tangency of the lines in Fig. 4, then the thickness of the ring perpendicular to its orbital plane is

$\delta z \approx \frac{1}{2}\left(\frac{\delta t}{P / 2 \pi}\right)^{2} a \sin \theta \approx \frac{1}{2}\left(\frac{\delta t}{P / 2 \pi}\right)^{2} \sin \beta_{\text {star }} \mathrm{AU}$,

where $a$ is the radius of the ring's orbit (assumed circular), $\beta_{\text {star }}$ is the star's Kepler-ecliptic latitude and $P \approx 1 \mathrm{y}$ is the orbital period of the platform (Earth or Kepler) from which observations are made. Because the ring's thickness determines the duration $\delta t$ it shadows the observer, $\delta t$ is independent of $A$.

The smallest estimate of $\delta z$ is obtained by taking $\delta t \sim$ $0.25 \mathrm{~d}$, the HWHM of the narrow dips. Then $\delta z \sim 10^{8} \mathrm{~cm}$. This is much less than the extension of the obscuring matter along its orbital direction, estimated (Sec. 3) as $\gtrsim 6 \times 10^{10}$ $\mathrm{cm}$ and proportional to only the first power of $\delta t$. That inequality is not surprising: longitudinal dispersion in velocity spreads a cloud of debris along its orbit to a length that increases until the orbit is filled, but transverse velocity dispersion only produces an oscillation with amplitude $\delta v P / 2 \pi$.

Larger estimates are possible. The interval between the

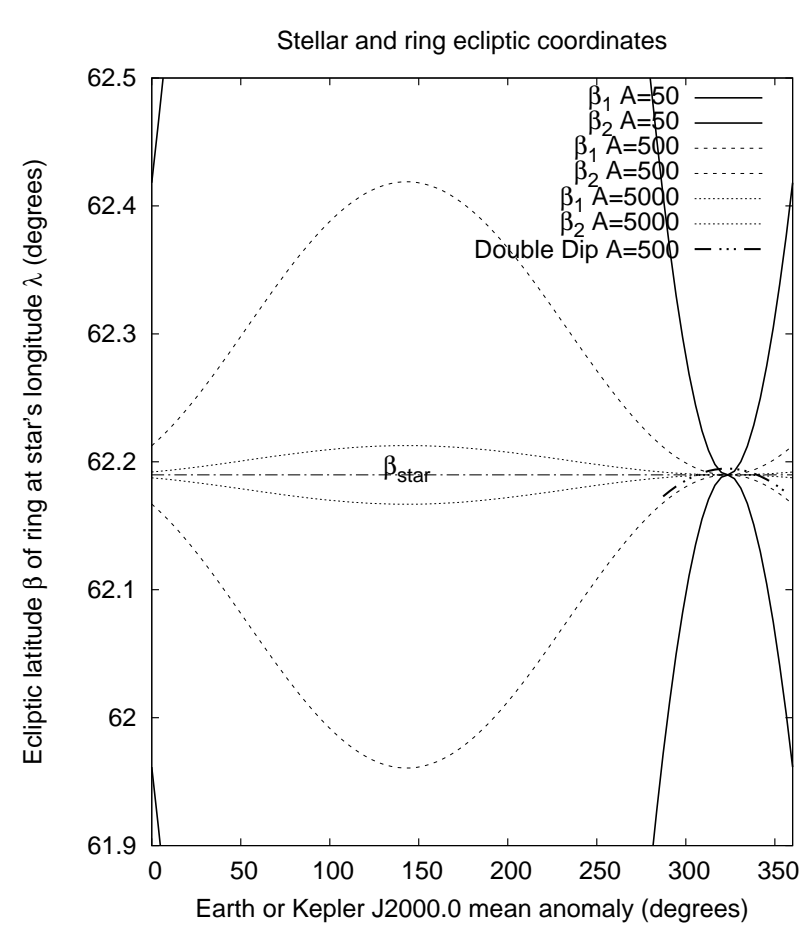

Figure 4. The ecliptic latitude $\beta$ of the ring at the right ascension of Boyajian's star as a function of the observer's orbital phase, assuming a circular ring and a circular orbit of the observer. These vary sinusoidally because of parallax. The two possible paths of a ring for each value of $A$ that maximize the time the ring is on the line of sight between observer and star are shown. A solution for $A=500$ that gives a double dip is also shown, with its small displacement from tangency exaggerated for clarity.

two deepest dips ( 5 and 8 in Boyajian et al. (2016)) is 726.86 $\mathrm{d}$, corresponding to two orbits of period of $363.43 \mathrm{~d}$ (the absence of a dip intermediate between these two may be explained if the ring is patchy; see discussion following Eq. 10). The discrepancy between this value and Kepler's orbital period, aside from requiring a patchy ring, indicates $\delta t \sim 5$ d. Then $\delta z \sim 5 \times 10^{10} \mathrm{~cm}$. If the three deep dips 8-10 are treated as a single event with $\delta t \sim 20$ d then the dilute envelope of weak absorption surrounding them, and the bundle of deep dips, are described by $\delta z \sim 10^{12} \mathrm{~cm}$.

The multiple distinct but closely spaced dips 8-10 and the deviation of the deep dips from accurate periodicity at Kepler's period indicate multiple rings with nearly identical inclinations to the Kepler ecliptic but slightly differing longitudes of their ascending nodes.

\section{DOUBLE DIP}

The apparent double minimum of dip 8 is suggestive of a ring whose positive excursion in $\beta$ is to a value slightly greater (or whose negative excursion is to a value slightly less) than that of Boyajian's star, so that after passing through the core of the ring the line of sight to the star passes through a lower column density of obscuring matter, then returns through the core and its longer path length and greater column density, before finally exiting the ring entirely. This 
is illustrated in Fig. 4. It is unsurprising that the deepest dip is doubled, implying passage (twice) of the line of sight through the central core of the ring, although with only four deep dips this is not statistically significant.

Analogously, shallower dips (the other numbered dips of Boyajian et al. (2016)) may result from lines of sight that approach but do not pass through the core of a ring, and that therefore have single minima. A single ring may produce such variations if it is not exactly planar, a result of perturbations by passing stars.

\section{RING MASS}

If the ring is uniformly filled with particles their mass may be estimated

$M \sim 2 \pi A \epsilon \kappa_{e f f}^{-1} \delta z \mathrm{AU}$,

where $\epsilon$ is the mean absorption averaged over the period over which the line of sight to the star passes through the ring and $\kappa_{\text {eff } f}$ is the effective mass extinction coefficient of ring particles (small for large particles) in $\mathrm{cm}^{2} / \mathrm{g}$. Estimating $\epsilon \sim 10^{-3}$ from the mean attenuation over $\delta t \sim 20 \mathrm{~d}$ if $\delta z \sim 10^{12}$ $\mathrm{cm}$ (Sec. 4) and taking $\kappa_{\text {eff }} \sim 10^{4} \mathrm{~cm}^{2} / \mathrm{g}$ (appropriate if ring particles are micron-sized) yields $M \sim 3 \times 10^{22} A_{5000} \mathrm{~g}$. Smaller $\delta z$ would imply larger $\epsilon \propto(\delta z)^{-1 / 2}$ but a smaller mass because the line of sight would spend less time passing through the ring. These values are consistent with the breakup of a $\sim 500 A_{5000}^{1 / 3} \mathrm{~km}$ diameter object into fine particles. Masses larger, in proportion to the particle size, would be required if the debris were coarser.

In the Kuiper belt $(A \sim 50)$ breakup of $\mathrm{a} \sim 100 \mathrm{~km}$ diameter body may be a plausible origin of a ring. At much greater $A$ a ring formed from primordial dust that never condensed into massive objects is more plausible, and would naturally consist of efficiently attenuating sub-micron particles.

\section{PRECESSION}

\subsection{Precession of the Ecliptic}

In the model proposed here a dip may occur when the ring is aligned to the direction to Boyajian's star to an accuracy of $\delta z /(A \mathrm{AU}) \approx 10^{-9} \delta z_{8} / A_{5000}$ radian, where $\delta z_{8} \equiv$ $\delta z / 10^{8} \mathrm{~cm}$. The plane of the ecliptic (and of Kepler's ecliptic) has a secular precesson rate $\approx 2 \times 10^{-6}$ radian/y (Dziobek 1892); periodic terms make this only a rough approximation. Hence an individual ring precesses out of alignment in a time

$t_{\text {misalign }} \sim \frac{1}{2} \times 10^{-3} \frac{\delta z_{8}}{A_{5000}} \mathrm{y}$.

\subsection{Precession of the Ring}

It is surprising, and possibly an argument against this model, that Boyajian's star is so far $\left(62^{\circ}\right)$ out of the ecliptic; the ring axis must make at least this angle with the ecliptic pole. Could this be explained by precession of the ring's orbit? I consider three sources of precessional torque: (i) The torque exerted by the concentration of mass towards the Galactic Center. This causes the ring's orbital axis to precess around the Galactic symmetry axis, in analogy to the precession of the Moon's orbit as a result of Solar torque, or of accretion discs in binary stars as a result of the torque exerted by the mass-losing star Katz (1973). Quantitative results depend on the distribution of mass in the Galaxy, but in general the ring precession rate is

$\Omega_{\text {pre }} \approx \Omega_{\text {Gal }} \frac{\Omega_{\text {Gal }}}{\Omega_{\text {ring }}} \sim 2 \pi \frac{A^{3 / 2}}{\left(2 \times 10^{8} \mathrm{y}\right)^{2}}$.

where $\Omega_{\text {Gal }}$ is the orbital angular velocity of the Solar System about the Galactic center and $\Omega_{\text {ring }}$ that of the ring about the Sun. The ring will have precessed at least one radian in the age of the Solar System if $A \gtrsim 10^{4}$. This is consistent with the upper end of the preceding estimates of A.

(ii) A fraction of the Galactic mass is concentrated in the disc with a density in the Solar neighborhood $\rho_{\text {disc }}$. Within this layer, the gravity gradient produces a precession rate

$\Omega_{\text {pre }} \approx \sqrt{G \rho_{d i s c}} \sqrt{\frac{\rho_{\text {disc }}(A \mathrm{AU})^{3}}{M_{\odot}}} \approx 2 \times 10^{-24} A^{3 / 2} \mathrm{~s}^{-1}$,

where $\rho_{\text {disc }} \approx 0.1 M_{\odot} \mathrm{pc}^{-3}$. The ring has precessed at least one radian in the age of the Solar System if $A \gtrsim 2 \times 10^{4}$, consistent with the upper end of the preceding estimates of A.

(iii) Random encounters with passing masses make the plane of the ring execute a random walk. A rough calculation leads to a diffusion coefficient

$$
\begin{aligned}
D_{\theta} & \sim \frac{G M_{s}}{b_{\min }^{2}} \frac{(A \mathrm{AU})^{3} n_{s}}{v_{s}} \frac{M_{s}}{M_{\odot}} \\
& \sim G \rho_{\text {disc }} \frac{A \mathrm{AU}}{v_{s}} \frac{M_{s}}{M_{\odot}} \sim 2 \times 10^{-24} \frac{M_{s}}{M_{\odot}} A \mathrm{~s}^{-1},
\end{aligned}
$$

where $M_{s}$ is the mass of the perturbing objects, $v_{s}$ their velocity, $n_{s}$ their number density, $\rho_{s}=M_{s} n_{s}$ and $b_{\text {min }}$ a lower cutoff on the impact parameter, taken as the ring orbital radius $A \mathrm{AU}$ (without this cutoff the integral over $b$ diverges, making $D_{\theta}$ ill-defined, and its effective value dependent on the single closest approach, therefore on the duration of the period of exposure to passing masses) and taking $\rho_{s}=0.1 M_{\odot} \mathrm{pc}^{-3}$ and $v_{s}=30 \mathrm{~km} / \mathrm{s}$. Then a 1 radian change of orientation requires a time

$t \sim \frac{1}{D_{\theta}} \sim 3 \times 10^{12} \frac{M_{\odot}}{M_{s}} \frac{1}{A_{5000}} \mathrm{y}$.

This process is negligible unless $M_{s} \gg M_{\odot}$, as might be the case if scattering is by clusters of stars or intermediate mass black holes.

Precession might explain the ring's inclination only if $A \gtrsim$ $10^{4}$ or if a significant fraction of the mass passing through the Galactic disc consists of objects of mass $\gtrsim 10^{3} M_{\odot} / A_{5000}$.

\section{STATISTICS}

Boyajian's star is apparently unique among the $\approx$ 150,000 stars studied by Kepler (Boyajian et al. 2016; Wright \& Sigurðsson 2016). Models that have an a priori probability of $10^{-6}$ (for example, require a uniformly distributed parameter to be within a fraction $10^{-6}$ of its range) 
may be excluded only at the $85 \%$ confidence level, so that they are acceptable by usual statistical criteria; those with an a priori probability of $10^{-7}$ may be excluded at the $98 \%$ confidence level but not at the $99 \%$ level. This differs from the familiar circumstance in which the brightest or firstdiscovered member of a class is studied, in which case demanding much higher a priori probability is appropriate. For Kepler objects, the class is the $\approx 150,000$ main sequence stars studied.

The Kepler field of view was $\Delta \theta \approx 0.2$ radian in each of $\beta$ and $\lambda$. It contains only one star like Boyajian's star, implying that the rings cover a fraction $\sim 10^{-5}$ of the field of view. Because rings cover narrow strips of nearly constant ecliptic latitude (with the small sinusoidal oscillation shown in Fig. 4), they must subtend a range $\delta \beta \sim 10^{-5} \Delta \theta \sim 2 \times$ $10^{-6}$ rad. Then their statistically inferred thickness $\delta z_{\text {stat }} \lesssim$ $A \delta \beta \mathrm{AU} \sim 10^{11} A_{5000} \mathrm{~cm}$. This is consistent with most of the estimates of Sec. 4, but the separations of dips 8-10 would require either that $A \sim 5 \times 10^{4}$ or that they each be produced by a separate narrow ring (or subring) in a broader bundle of rings with similar, but not identical, orbits. The width of the bundle $\delta z_{\text {bundle }} \sim 5 \times 10^{10}-10^{12} \mathrm{~cm} \gg \delta z$. The wider shallow dips would be the result of a much broader (but much less opaque) distribution of matter within this bundle.

We can reject the hypothesis that the rings producing deep dips are unrelated. To explain dips 8-10 several very narrow rings must each be oriented to produce deep dips in one star (Boyajian's) in the Kepler field with individual $a$ priori probability $p=\mathcal{O}(1 / 150,000)$, but none in any other of the 150,000 stars in the field. If the orientations, separated by $\sim \delta z_{\text {bundle }} \gtrsim \delta z_{\text {stat }}$, are uncorrelated, the probability that $n$ rings are oriented to produce dips in any one star but none in any other star is given by the Poisson distribution for $n$ events with mean probability $n p \ll 1$, and is negligible. Hence the rings producing dips must be correlated-part of a single bundle with $\delta z_{\text {bundle }} \lesssim \delta z_{\text {stat }}$.

I suggest two possible explanations:

(i) $A \gtrsim 5000$. Then $\delta z_{\text {stat }} \sim A \delta \beta \mathrm{AU} \sim 10^{11} A_{5000} \mathrm{~cm}$, so that $\delta z_{\text {bundle }} \lesssim \delta z_{\text {stat }}$. The rings are far beyond the Kuiper belt, and the entire bundle is so narrowly confined in $\beta$ that they intercept the light of only one star in the Kepler field.

(ii) Obscuring matter is concentrated in a narrow range of $\lambda$ (equivalently, true or mean anomaly). Then, while a fraction $f_{\beta} \sim 10^{11} \mathrm{~cm} /(A \Delta \theta \mathrm{AU}) \sim 10^{-5} / A_{5000}$ of the Kepler stars have the right $\beta$ to be occulted, only a fraction $f_{\lambda}=\delta \lambda / \Delta \theta$ also have the right $\lambda$; the requirement that the Kepler field contain only $\mathcal{O}(1)$ star like Boyajian's star implies $f_{\beta} f_{\lambda} \sim 10^{-5}$

\section{PREDICTIONS}

If particulate rings explain the dips of Boyajian's star, some predictions can be made:

(i) Dips may be observed from the Earth when a ring grazes the line of sight, as shown in Fig. 4. This will occur with a period $P_{E}=365.25 \mathrm{~d}$. Because $P_{K} \neq P_{E}$, the times of dips observed by Kepler must be extrapolated back to its launch from Earth; the deep dip 5 occurred 728 days from launch, when Kepler was 16 days behind Earth in its Earthtrailing orbit. Allowing for that, we predict that dips might be observed from Earth around

$\mathrm{BJD}=2455609+365.25 n$,

where $n=1,2, \ldots$, although the apparent precision is not justified by the observed scatter of dip timing.

For Kepler data $365.25 \mathrm{~d}$ should be replaced by the Kepler year of $372.53 \mathrm{~d}$. Several shallower dips (1, 2, 3 and 6 of Boyajian et al. (2016) and a dip on May 19, 2017 ( Boyajian et al. 2017)) do not fit this pattern. This may be attributable to a complex ring structure with several less opaque sub-rings. Shallow dip 4 of Boyajian et al. (2016) is $366 \mathrm{~d}$ from deep dip 5 , in reasonable agreement.

The alignment of narrow rings, such as those inferred to produce deep dips, with the star lasts less than a year (Sec. 7.1), so that annual recurrences are not expected. For a ring complex producing extended shallow absorption $\delta z \sim 10^{12} \mathrm{~cm}$ (Sec. 4) and repetitions may continue for many years. Such a complex may contain narrow rings capable of producing deep dips, like dips 8-10 of Boyajian et al. (2016), that have much smaller $\delta z$ and will not individually repeat.

(ii) The rays from the star to a point observer in the inner Solar System are spread over a width and height $\ell_{S S} \approx 60 A_{5000} \mathrm{~km}$ at the ring, traversed by the observer's motion in $t_{S S}=\ell_{S S} / v_{\text {orb }} \sim 2 A_{5000} \mathrm{~s}$. The observed flux is an average over finer structure, but ring structure on scales $\gtrsim \ell_{S S}$, if present, might be observed as intensity fluctuations in sufficiently high cadence observations.

Photometry will show time lags between terrestrial and deep space (from future Kepler-like platforms in orbits trailing or in advance of the Earth) observations equal to their separation along Earth's direction of orbital motion divided by $v_{\text {orb }}$.

The rings have structure on scales $\delta z$ normal to the observer's motion. Dips observed from sites separated by distances $\gtrsim \delta z$ in that direction will be uncorrelated. Because $\delta z$ may be $\sim 10^{8} \mathrm{~cm}$, smaller than the size of the Earth (Sec. 4), it may be possible to observe this decorrelation between terrestrial sites.

In contrast, in circumstellar models of the dips observers anywhere in the Solar System see the same variations, aside from small delays attributable to light propagation time.

(iii) Kepler's orbit is inclined to the ecliptic by $i=0.45^{\circ}$ (Borucki, et al. 2010; Koch, et al. 2010). As a result, a very narrow ring that produces dips at the Kepler spacecraft would not produce dips at Earth, and vice versa. The outof-plane separation of these orbits $\delta z_{\text {orb }}=a \sin i \sin \chi$, where $\chi$ is the angular separation of Earth and Kepler measured from the Sun. For dips $8-10 \chi \approx 0.52 \mathrm{rad}$, implying $\delta z_{\text {orb }} \approx$ $6 \times 10^{10} \mathrm{~cm}$. If the ring is thicker than this (as suggested in Sec. 4) then the same dips could have been observed from Earth. Even for the maximum value of $|\sin \chi|=1$, $\delta z_{\text {orb }} \approx 1.2 \times 10^{11} \mathrm{~cm}$, and for plausible $\delta z>\delta z_{\text {orb }}$ the Kepler dip timing (allowing for the differing orbital periods) may be extrapolated to dips observable from Earth.

\section{DISCUSSION}

The occurrence of deep dips in two epochs separated by about two Kepler-years is a hint that the phenomenon may be local rather than circumstellar. This evidence is suggestive but not statistically compelling because the interval dif- 
fers from an exact integer multiple of Kepler-years by a few percent. However, the difficulty of developing a persuasive circumstellar model and the history of discovery of narrow planetary rings by stellar occultation justify investigation of possible explanations involving Solar System rings. Preliminary estimates indicate this model is not absurd.

It is possible to constrain the time $T$ since the event that created the particles if they consist of single clouds of debris moving on Keplerian orbits since their formation, as would be plausible if they consist of dust that never condensed to a massive object. Assuming a width $\delta A=\delta z / A$ :

$\delta \lambda \sim \frac{3}{2} \frac{\delta z}{A \mathrm{AU}} A^{-3 / 2} \frac{2 \pi T}{\mathrm{y}} \mathrm{rad}$.

Inverting,

$T \sim 4 \times 10^{9} \frac{f_{\lambda}}{0.01} \frac{10^{9} \mathrm{~cm}}{\delta z} A_{5000}^{5 / 2} \mathrm{y}$.

After times $\gtrsim T$ the matter spreads into a continuous ring, but until that happens it may be clumpy, as implied by the fact that some expected dips are missing, such as one between dips 5 and 8. For larger values of $A\left(A_{5000} \gtrsim 1\right)$ $T$ may exceed the age of the Solar System, explaining the survival of patchy structure.

Matter may be distributed very nonuniformly along a ring, so that in any Kepler-year the line of sight need not intersect a significant quantity of absorbing matter during the brief period when the line of sight grazes the ring. This can explain the absence of deep dips between dip 5 and the cluster of dips $8-10$ or a Kepler-year prior to dip 5. There may be more potential "Boyajian's Stars" among the stars observed by Kepler that did not dip during its four years of observation because they were viewed through gaps in a ring. Eq. 8 is a necessary but not sufficient condition for the observation of dips in KIC 8462852 from a ring that produced dips 4, 5 and 8-10.

This model makes predictions as to the timing of possible (but only possible, because the ring appears to be patchy, future dips produced by the ring that produced dips 5 and $8-10$. The existence of other rings, producing dips at other times, is not excluded. There may be a finite, and perhaps short, correlation length between dips observed from separate locations. Testing this latter prediction would provide a clear distinction between circumstellar and Solar System models (independent of the specific ring model proposed here). The observation of decorrelation between Solar System observers would definitively prove a Solar System origin, but to disprove it would require observations from at least one distant $(\gtrsim 0.01 \mathrm{AU})$ space platform as well as from Earth.

Ring particles move $2 \pi A^{-1 / 2} \mathrm{AU} \sim A_{5000}^{-1 / 2} 10^{12} \mathrm{~cm}$ in a year (or Kepler year), so that each year the line of sight samples an independent part of the ring, even on the penumbral scale $\sim 10^{12} \mathrm{~cm}$ estimated in Sec. 3. Dips need not repeat every year.

Observations with higher cadence than Kepler's may reveal non-tangential passages through a ring that last $\delta z /\left(v_{\text {orb }}|\sin \Delta \beta|\right)$, where $\Delta \beta$ is the difference between the ecliptic latitude $\beta$ of the ring and the star. This can be as short as $\sim 30 \mathrm{~s}$ for $\delta z \sim 10^{8} \mathrm{~cm}$. Non-tangential passages might also appear in the Kepler database as shallow, singledatum, dips. For stars in the Kepler field $|\Delta \beta| \leq 0.2 \mathrm{rad}$, and durations would be at least a few minutes. The absence of high frequency noise (Sec. 2) indicates that such effects are small.

This model offers no natural explanation of the steady dimming reported by Montet \& Simon (2016). It might be explained as a consequence of a broader ring of dilute obscuring material, but that adds an ad hoc complexity to the model.

\section{ACKNOWLEDGEMENTS}

I thank D. Palmer, S. Phinney and S. A. Rappaport for useful discussions on every aspect of this problem and an anonymous referee for much constructive criticism. This research has made use of the NASA Exoplanet Archive, which is operated by the California Institute of Technology, under contract with the National Aeronautics and Space Administration under the Exoplanet Exploration Program.

\section{REFERENCES}

Borucki, W. J. et al. 2010 Science 327, 977.

Boyajian, T. S. et al. 2016 MNRAS 457, 3988.

Boyajian, T. S. et al. 2017 ATEL 10405.

Dyson, F. J. 1992 QJRAS 33, 45.

Dziobek, O. 1892 Mathematical Theories of Planetary Motions, Register Publishing (Ann Arbor, Mich.) p. 294 https://en.wikipedia.org/wiki/Ecliptic accessed June 28, 2017.

Elliot, J. L., Dunham, E. \& Mink, D. 1977 Nature 267, 328.

Katz, J. I. 1973 Nat. Phys. Sci. 246, 87.

Koch, D. G., et al. 2010 ApJ 713, L79.

Montet, B. T. \& Simon, J. D. 2016 ApJ 830, L39.

Wright, J. T. \& Sigurðsson, S. 2016 ApJ 829, L3.

This paper has been typeset from a $\mathrm{T}_{\mathrm{E}} \mathrm{X} / \mathrm{L}_{\mathrm{A}} \mathrm{T}_{\mathrm{E}} \mathrm{X}$ file prepared by the author. 